Tanja Vogler

Das politische Subjekt des queeren Aktivismus

Queer Studies | Band 32 
Die freie Verfügbarkeit der E-Book-Ausgabe dieser Publikation wurde ermöglicht durch POLLUX - Informationsdienst Politikwissenschaft

POLLUXX) Informationsolingst

und der Open Library Community Politik 2022 - einem Netzwerk wissenschaftlicher Bibliotheken zur Förderung von Open Access in den Sozial- und Geisteswissenschaften:

Vollsponsoren: Freie Universität Berlin Universitätsbibliothek | Staatsbibliothek zu Berlin | Universitätsbibliothek der HumboldtUniversität zu Berlin | Universitätsbibliothek Bielefeld | Universitätsbibliothek der Ruhr-Universität Bochum | Universitäts- und Landesbibliothek Bonn | Staats- und Universitätsbibliothek Bremen | Universitäts- und Landesbibliothek Darmstadt | Sächsische Landesbibliothek Staats- und Universitätsbibliothek Dresden (SLUB) | Universitäts- und Landesbibliothek Düsseldorf | Universitätsbibliothek Frankfurt am Main | Justus-Liebig-Universität Gießen | Niedersächsische Staats- und Universitätsbibliothek Göttingen | Universitätsbibliothek der FernUniversität in Hagen | Staats- und Universitätsbibliothek Carl von Ossietzky, Hamburg | Gottfried Wilhelm Leibniz Bibliothek - Niedersächsische Landesbibliothek | Technische Informationsbibliothek (TIB Hannover) | Universitätsbibliothek Kassel | Universitätsbibliothek Kiel (CAU) | Universitätsbibliothek Koblenz · Landau | Universitätsund Stadtbibliothek Köln | Universitätsbibliothek Leipzig | Universitätsbibliothek Marburg | Universitätsbibliothek der Ludwig-Maxi-
milians-Universität München | Max Planck Digital Library (MPDL) | Universität der Bundeswehr München | Universitäts- und Landesbibliothek Münster | Universitätsbibliothek Erlangen-Nürnberg |Bibliotheks- und Informationssystem der Carl von Ossietzky Universität Oldenburg | Universitätsbibliothek Osnabrück | Universitätsbibliothek Passau | Universitätsbibliothek Vechta | Universitätsbibliothek Wuppertal | Vorarlberger Landesbibliothek | Universität Wien Bibliotheks- und Archivwesen | Zentral- und Hochschulbibliothek Luzern | Universitätsbibliothek St. Gallen | Zentralbibliothek Zürich

Sponsoring Light: Bundesministerium der Verteidigung | ifa (Institut für Auslandsbeziehungen), Bibliothek | Landesbibliothek Oldenburg | Ostbayerische Technische Hochschule Regensburg, Hochschulbibliothek | ZHAW Zürcher Hochschule für Angewandte Wissenschaften, Hochschulbibliothek

Mikrosponsoring: Stiftung Wissenschaft und Politik (SWP) - Deutsches Institut für Internationale Politik und Sicherheit | Leibniz-Institut für Europäische Geschichte

Tanja Vogler (PhD), geb. 1989, lehrt und forscht an der Schnittstelle von Gender und Medizin an der Medizinischen Universität Innsbruck. Sie war Mitglied des Doktoratskollegs Geschlecht und Geschlechterverhältnisse in Transformation« des Centers Interdisziplinäre Geschlechterforschung Innsbruck und promovierte zum queeren Aktivismus. Für ihre Dissertation »Das politische Subjekt des queeren Aktivismus. Diskurs- und Akteurskonstellationen queerer Politiken im deutschsprachigen Raum: Eine empirische Untersuchung « erhielt sie den Gabriele-Possanner Förderungspreis 2021 des Bundesministerium Bildung, Wissenschaft und Forschung sowie den GenderFemPreis 2021 der Leopold-Franzes-Universität Innsbruck. Ihre Forschungsschwerpunkte liegen im Bereich der Gender, Queer und Discourse Studies. 


\section{Tanja Vogler}

\section{Das politische Subjekt des queeren Aktivismus}

Diskurs- und Akteurskonstellationen queerer Politiken

im deutschsprachigen Raum 
Dissertation am Institut für Erziehungswissenschaft der Leopold-Franzens-Universität Innsbruck (Einreichung 2020, Disputation 2021).

Erstgutachterin/Erstbetreuerin: Univ.-Prof.'in Dr. Michaela Ralser

Zweitgutachterin: Univ.-Prof.'in Dr. Bettina Kleiner

Zweitbetreuerin: Univ.-Prof.'in Dr. Susanne Maurer

Ich danke dem Studienwerk der Heinrich-Böll-Stiftung, das die Arbeit von April 2017 bis September 2019 mit einem Stipendium gefördert hat.

Diese Publikation wurde mit freundlicher Unterstützung und finanzieller Förderung durch nachfolgende Institutionen der Leopold-Franzens-Universität Innsbruck veröffentlicht: Vizerektorat für Forschung, Fakultät für Bildungswissenschaften, Institut für Erziehungswissenschaft, Forschungsplattform Center Interdisziplinäre Geschlechterforschung und Doktoratskolleg Geschlecht Geschlechterverhältnisse in Transformation: Räume - Relationen - Repräsentationen.

\begin{tabular}{|c|c|c|c|}
\hline $\begin{array}{l}\text { universität } \\
\text { innsbruck }\end{array}$ & $\begin{array}{l}\text { universität } \\
\text { innsbruck }\end{array}$ & $\begin{array}{l}\text { universität } \\
\text { innsbruck }\end{array}$ & $\begin{array}{l}\text { universität } \\
\text { innsbruck }\end{array}$ \\
\hline $\begin{array}{l}\text { Büro der Vizerektorin } \\
\text { für Forschung }\end{array}$ & $\begin{array}{l}\text { Fakultät für } \\
\text { Bildungswissenschaften }\end{array}$ & $\begin{array}{l}\text { Institut für } \\
\text { Erziehungswissenschaft }\end{array}$ & $\begin{array}{l}\text { Forschungsplattform Center Interdisziplinäre } \\
\text { Geschlechterforschung Innsbruck }\end{array}$ \\
\hline
\end{tabular}

\section{Bibliografische Information der Deutschen Nationalbibliothek}

Die Deutsche Nationalbibliothek verzeichnet diese Publikation in der Deutschen Nationalbibliografie; detaillierte bibliografische Daten sind im Internet über http://dnb.d-nb.de abrufbar.

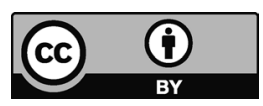

Dieses Werk ist lizenziert unter der Creative Commons Attribution 4.0 Lizenz (BY). Diese Lizenz erlaubt unter Voraussetzung der Namensnennung des Urhebers die Bearbeitung, Vervielfältigung und Verbreitung des Materials in jedem Format oder Medium für beliebige Zwecke, auch kommerziell. (Lizenztext: https://creativecommons.org/licenses/by/4.o/deed.de)

Die Bedingungen der Creative-Commons-Lizenz gelten nur für Originalmaterial. Die Wiederverwendung von Material aus anderen Quellen (gekennzeichnet mit Quellenangabe) wie z.B. Schaubilder, Abbildungen, Fotos und Textauszüge erfordert ggf. weitere Nutzungsgenehmigungen durch den jeweiligen Rechteinhaber.

\section{Erschienen 2022 im transcript Verlag, Bielefeld (c) Tanja Vogler}

Umschlaggestaltung: Maria Arndt, Bielefeld

Umschlagabbildung: Street Transvestite Action Revolutionaries at the Christopher Street Liberation Day March, 24.06.1973 by Leonard Fink Photographs; License: The Lesbian, Gay, Bisexual \& Transgender Community Center

Korrektorat: Dr.in Margret Haider; Dr.in Marion Bach

Druck: Majuskel Medienproduktion $\mathrm{GmbH}$, Wetzlar

Print-ISBN 978-3-8376-6083-8

PDF-ISBN 978-3-8394-6083-2

EPUB-ISBN 978-3-7328-6083-8

https://doi.org/10.14361/9783839460832

Buchreihen-ISSN: 2703-1365

Buchreihen-eISSN: 2703-1373

Gedruckt auf alterungsbeständigem Papier mit chlorfrei gebleichtem Zellstoff.

Besuchen Sie uns im Internet: $h t t p s: / / w w w . t r a n s c r i p t-v e r l a g . d e$

Unsere aktuelle Vorschau finden Sie unter www.transcript-verlag.de/vorschau-download 